Portland State University

PDXScholar

$1-1-2010$

\title{
Characterization and correction of dark current in compact consumer cameras
}

\author{
Justin Charles Dunlap \\ Portland State University \\ Erik Bodegom \\ Portland State University \\ Ralf Widenhorn \\ Portland State University
}

Follow this and additional works at: https://pdxscholar.library.pdx.edu/phy_fac

Part of the Physics Commons

Let us know how access to this document benefits you.

\section{Citation Details}

Justin C. Dunlap ; Erik Bodegom ; Ralf Widenhorn; Characterization and correction of dark current in compact consumer cameras. Proc. SPIE 7536, Sensors, Cameras, and Systems for Industrial/Scientific Applications XI, 75360J (January 25, 2010)

This Article is brought to you for free and open access. It has been accepted for inclusion in Physics Faculty Publications and Presentations by an authorized administrator of PDXScholar. Please contact us if we can make this document more accessible: pdxscholar@pdx.edu. 


\title{
Characterization and correction of dark current in compact consumer cameras
}

\author{
Justin C. Dunlap, Erik Bodegom, and Ralf Widenhorn \\ Portland State, Portland, OR 97207
}

\begin{abstract}
A study of dark current in digital imagers within consumer grade digital cameras is presented. Dark current is shown to vary with temperature, exposure time, and ISO setting. Further, dark current is shown to increase in successive images during a series of images. Consumer cameras are often designed to be as compact as possible and therefore the digital imagers within the camera frame are prone to heat generated by nearby elements within the camera body. It is the scope of this work to characterize the dark current in such cameras and to show that the dark current, in part due to heat generated by the camera itself, can be corrected for by using hot pixels on the imager. This method generates computed dark frames based on the dark current indicator value of the hottest pixels on the chip. We compare this method to standard methods of dark current correction.
\end{abstract}

Keywords: Dark current, image correction, digital imagers, CCD, CMOS

\section{INTRODUCTION}

We have previously shown that noise generated by thermally excited electrons, so called dark noise, in digital imagers can be corrected by analyzing the pixels that show the greatest amounts of dark current. This method has shown to work in general for Charge-Coupled Devices ${ }^{1}$ (CCDs) and Complimentary Metal-Oxide Semiconductor (CMOS) imagers, ${ }^{2}$ as well as specifically for digital imagers contained within DSLR cameras. ${ }^{3}$ DSLR cameras are generally considered to be higher quality consumer cameras. This work extends the study of using this method to analyze and correct dark current in compact, generally less expensive, consumer cameras.

The very attributes that define this class of camera, their affordability and compact size, are likely indicators that dark noise is a greater issue in this class of camera. Dark noise is caused by defects and impurities within the silicon of the pixel. ${ }^{4,5,6}$ The price point of compact consumer cameras would seem to indicate that the best digital imagers, in terms of lowest impurities and therefore lowest dark noise, would be selectively chosen for more expensive, higher-end, cameras. In addition, it was found when examining DSLR imagers that the confinement of the imager within the camera body significantly affected the dark current. That is, heat generated by the camera itself heated the imager and created more dark noise for subsequent images. Consumer cameras are generally not cooled and therefore heat will be trapped until it can dissipate through the camera body; this was found to take at least a few minutes for the DSLR cameras we studied. Compact consumer cameras have the different electronic elements of the camera confined to an even smaller volume suggesting a greater influence of heat generated by the various elements on the imager, and therefore also a greater effect on dark noise.

To accurately study the dark noise in consumer cameras, it is necessary to obtain images that have not been compressed nor processed. The typical file generated by consumer cameras is in the JPEG format, a format that allows for variable levels of compression. However, the compression is not loss-less and some information, including some of the dark noise signal, is lost in the compression routines. In addition many cameras will process the information from the imager, such as by sharpening or color correcting the image, in an effort to make the image more aesthetically pleasing. These processing routines further obfuscate the original signal from the imager and further mask the dark noise. Therefore, we used an open-source software, called CHDK, ${ }^{7}$ designed to be installed on Canon cameras that allow obtaining uncompressed, unprocessed, images in the RAW file format. In addition, CHDK also allows for long exposure time control. Unfortunately, this limited the study to one manufacturer of cameras, Canon.

Sensors, Cameras, and Systems for Industrial/Scientific Applications XI, edited by Erik Bodegom,

Valérie Nguyen, Proc. of SPIE-IS\&T Electronic Imaging, SPIE Vol. 7536, 75360J · @ 2010

SPIE-IS\&T · CCC code: 0277-786X/10/\$18 - doi: 10.1117/12.840440 
Compact consumer cameras do correct for dark noise, however the methods they use limit the consumer to irreversible processing done by the camera that may not be the best possible means of correction. The traditional method for correction done on a long exposure-time image involves taking a single dark frame with the same settings as the light exposure immediately after the light exposure. This not only takes twice the time, but it also introduces dark current shot noise, as well as read noise, and, as we will show, does not do as good of a job at dark correction as the computed dark frames. In addition, built up heat from camera elements, including the image sensor itself, exasperated due to the compact nature of these cameras, change the internal temperature of the camera with use, and therefore the dark frame will not be taken with the same conditions as the light exposure.

\section{DARK CURRENT BEHAVIOR}

Data is presented for three compact consumer grade cameras. Images were taken with a Canon PowerShot SD1000, a Canon S2IS, and a Canon PowerShot A570 IS. These cameras all generate images with a 10-bit pixel depth. The images were taken in an environment that was sealed from light and temperature controlled.

\subsection{Canon PowerShot SD1000}

The SD1000, announced February 22, 2007, has a 1/2.5" type CCD sensor (approximately $5.7 \mathrm{~mm} \times 4.3 \mathrm{~mm}$ ), with 7.1 million effective pixels in a grid of $3072 \times 2304$ pixels. To gauge the dark current response due to exposure time, we took ten images for each exposure time of 1, 5, 10, 20, 30, 40,50 and $60 \mathrm{~s}$, with the $1 \mathrm{~s}$ set taken first and ascending to $60 \mathrm{~s}$. The camera was set to an ISO setting of 400 and the external temperature within the enclosure was $24{ }^{\circ} \mathrm{C}$. The average was taken for each set of images after discarding the maximum and minimum values for each pixel. Figure 1 shows the exposure time dependence for eight representative pixels on the imager. For each pixel, the pixel coordinates are given in parentheses. Pixels 1 through 6 are all hot pixels to varying degrees while Pixel 7 and 8 represent a more typical pixel on the imager that has a smaller dark current. The pixels show an increase in dark noise with increasing exposure time with fairly linear responses except for when the pixels begin to approach the saturation level of 1023 counts.

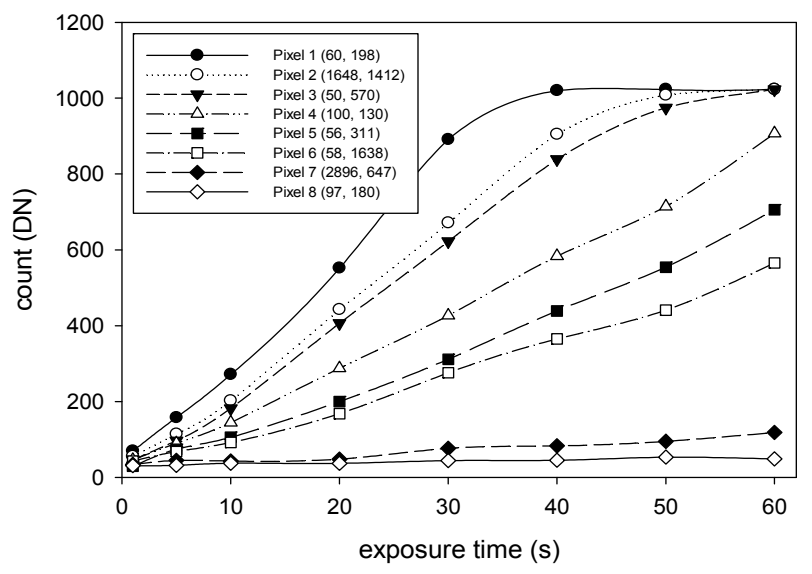

Fig. 1. Exposure time dependence of dark current for eight pixels, taken at ISO $=400$ and $24{ }^{\circ} \mathrm{C}$.

Figure 2 shows the ISO setting dependence and Fig. 3 shows the temperature dependence of dark current for the same eight pixels as displayed in Fig. 1. Again, each data point represents the average of ten frames with the maximum and minimum values for each pixel removed. Despite the claim by Canon that ISO sensitivity of the SD1000 goes up to 1600, it was found that the dark current does not change past a setting of 800 and deviates from a linear dependence after a setting of 400 . This may be due to additional processing done by the Canon software to obtain effective ISO sensitivities past 400 that is therefore not seen in this data due to using the CHDK software. Regardless, the dark current does show a linear and predictable response for most of the ISO range. Likewise, dark 
current response versus temperature appears to be predictable until values near saturation for the pixels in the imager as shown for the eight pixels presented in Fig. 3.

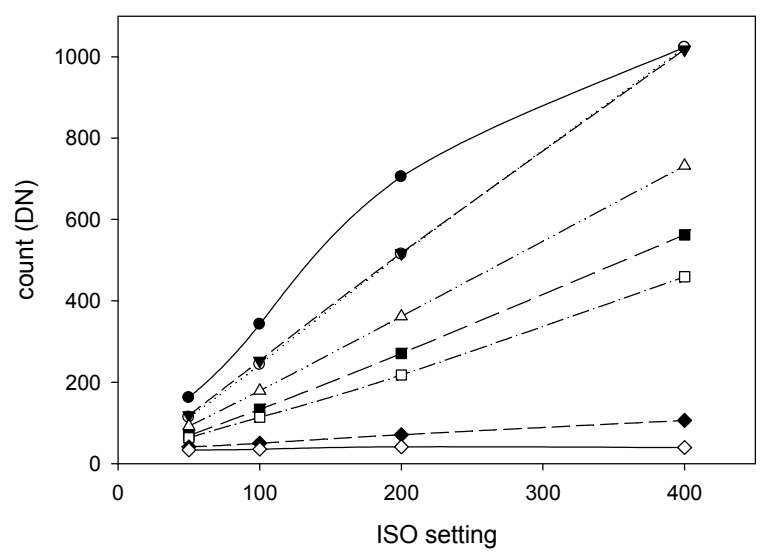

Fig. 2. Dark current dependence on ISO setting for eight pixels. Taken at $24{ }^{\circ} \mathrm{C}$ with $60 \mathrm{~s}$ exposure times. (Same pixels as shown in Fig. 1.)

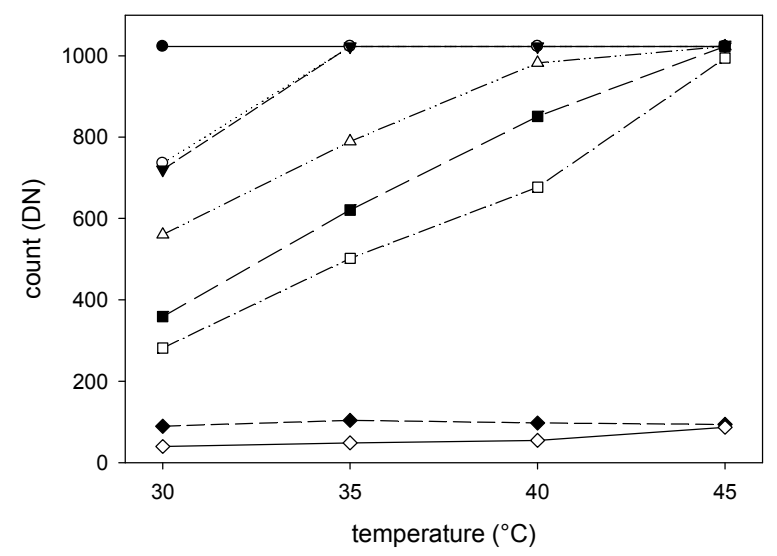

Fig. 3. Dependence of dark current for eight pixels on external temperature. Taken with ISO $=400$ and an exposure time of $50 \mathrm{~s}$. (Same pixels as shown in Fig. 1.)

It was found for DSLR cameras that frame number also played a significant role in the increase of dark current when multiple images were taken in succession. To ascertain this effect on compact cameras, a set of 50 frames were taken with the SD1000 at an ISO setting of 400, an integration time of $15 \mathrm{~s}$, and with a $60 \mathrm{~s}$ rest between frames. The temperature within the enclosure increased from $23.8^{\circ} \mathrm{C}$ during the first frame to $24.3^{\circ} \mathrm{C}$ during the last frame. Figure 4 shows the response of eight pixels on the imager with these settings. Even with a rest time four times greater than the integration time, the dark current increases significantly from the first frame to the fiftieth frame. As an example, Pixel 1 nearly doubled, increasing from 196 counts in the first frame to 342 counts in the last frame, a relative increase of $74 \%$. This increase likely cannot be explained by the external temperature change of $0.5^{\circ} \mathrm{C}$ alone.

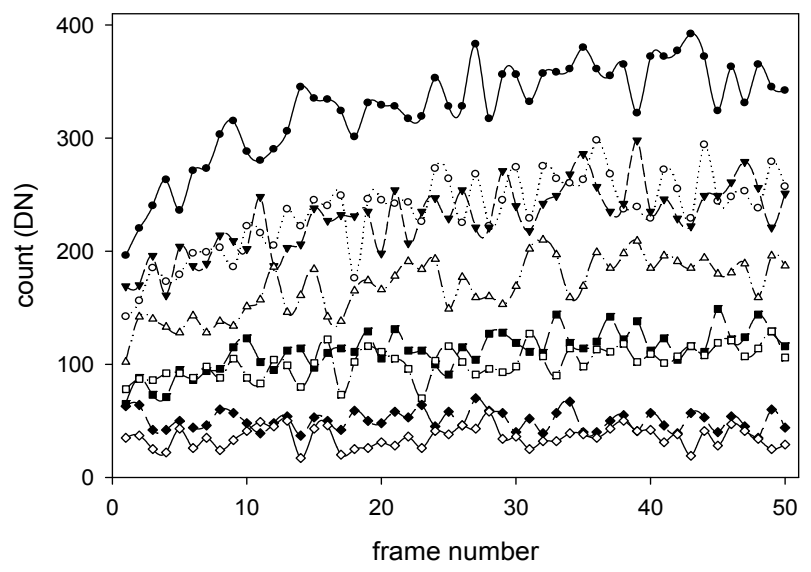

Fig. 4. Dark current dependence on frame number for eight pixels, taken at ISO $=400,24{ }^{\circ} \mathrm{C}$, integration times of $15 \mathrm{~s}$ with a rest period of $60 \mathrm{~s}$ between frames. (Same pixels as shown in Fig. 1.)

\subsection{Canon S2IS}

The S2IS, announced April 22, 2005, has a 1/2.5" type CCD sensor, with 5.0 million effective pixels in a grid of 2592 x 1944 pixels. Figure 5 shows the S2IS' dark current response for varying exposure times (Fig. 5a), varying temperatures (Fig. 5b), and varying ISO setting (Fig. 5c). To determine exposure time dependence 10 pictures were 
taken at various exposure times allowed by the CHDK software, namely at 2, 4, 8, 16, 32, 51 and $62 \mathrm{~s}$, with an external temperature of $25^{\circ} \mathrm{C}$ and at an ISO setting of 200. Images taken at $62 \mathrm{~s}$ consistently showed no significant change from those taken at $51 \mathrm{~s}$ and may be due to CHDK incorrectly allowing for exposure times longer than those allowed by the camera hardware. Therefore, values for the $62 \mathrm{~s}$ exposures were not shown in the graph displaying dark current dependence on exposure time for eight representative pixels shown in Fig. 5a. Pixels 1 through 6 all show significant increases in dark current with exposure time and would make good candidates for dark current indicators on this imager. The pixels show an increase in dark current with exposure time, with a positive curvature likely due to increasing internal temperatures of the imager with increasing exposures as the frames with longer exposure times were taken after those with shorter exposure times. Pixels 7 and 8 are chosen to represent pixels with little dark current and are representative of a typical pixel on the imager. To test the dependence on temperature, 10 images were taken at $25,30,35,40$, and $45^{\circ} \mathrm{C}$, with exposure times of $51 \mathrm{~s}$, and at an ISO setting of 200 . The same eight pixels' dark current dependence on temperature are presented in Fig. 5b, where again Pixels 1 through 6 show a large amount of dark current and a predictable response of dark current to increasing temperature. However, Pixels 1 through 3 saturate and therefore would not make ideal indicators in this regime. To test the dependence on ISO setting, 10 images were taken at ISO settings of 50,100, 200,400,500,600, 700, and 800, with exposure times of $51 \mathrm{~s}$ and at a temperature of $25^{\circ} \mathrm{C}$. There appears to be no change in dark current for all pixels after an ISO setting of half of the Canon advertised maximum ISO of 400 (ISO=200). Regardless, all pixels do show a linear increase in dark current with ISO setting up to 200 and therefore would have predictable dark current behavior in this regime with Pixels 1 through 6 being possible dark current indicators.
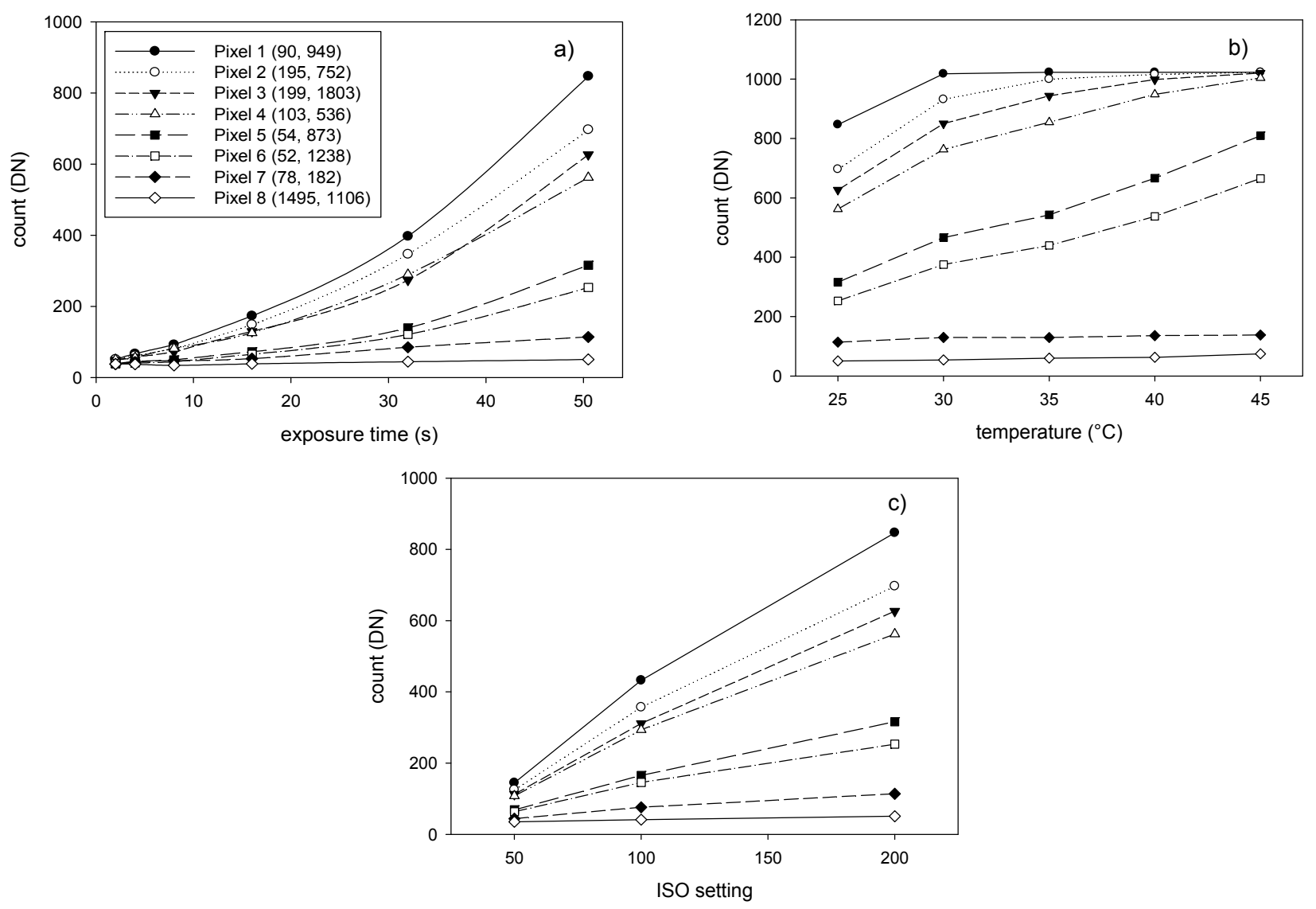

Fig. 5. Dependence of dark current for eight Canon S2IS pixels. a) dependence on exposure time. Taken at $25^{\circ} \mathrm{C}$ with $\mathrm{ISO}=200 . \mathrm{b}$ ) dependence on temperature. Taken at ISO=200 with an exposure time of $51 \mathrm{~s}$. c) dependence on ISO setting. Taken at $25^{\circ} \mathrm{C}$ with an exposure time of $51 \mathrm{~s}$. 


\subsection{Canon PowerShot A570 IS}

The A570 IS, also announced February 22, 2007, has a 1/2.5" type CCD sensor, with 7.1 million effective pixels in a grid of $3072 \times 2304$ pixels. Figure 6 shows the dependence of dark current for eight of the A570's pixels on exposure time (Fig. 6a) and on ISO setting (Fig. 6b). To test the dependence on exposure time, ten frames each were taken at exposure times of $1,5,10,20,32$, and $64 \mathrm{~s}$, with an ISO setting of 400 , and at a temperature of $24^{\circ} \mathrm{C}$. All eight pixels show a linear increase in dark current with exposure time, except for Pixel 1 which saturates after the $32 \mathrm{~s}$ exposure. A typical pixel on the A570 is represented by Pixel 8, which has less dark current but its dark current still appreciably increases with exposure time. To test dependence on ISO setting, 10 frames each were taken at ISO settings of $40,80,100,200,300,400,800$, and 1600 , at a temperature of $24^{\circ} \mathrm{C}$, and with exposure times of $32 \mathrm{~s}$. All eight pixels' dark current show a linear increase with ISO setting until reaching a setting of 400 as shown in Fig. 6 b. Responses past this setting did not give the expected increase in dark current and showed no change for settings larger than half the claimed maximum ISO setting of 1600. The A570 proved to have significantly more hot pixels than the other two cameras, with even the typical pixels, such as Pixel 8, showing a steady and appreciable increase in dark current with exposure time and ISO setting.
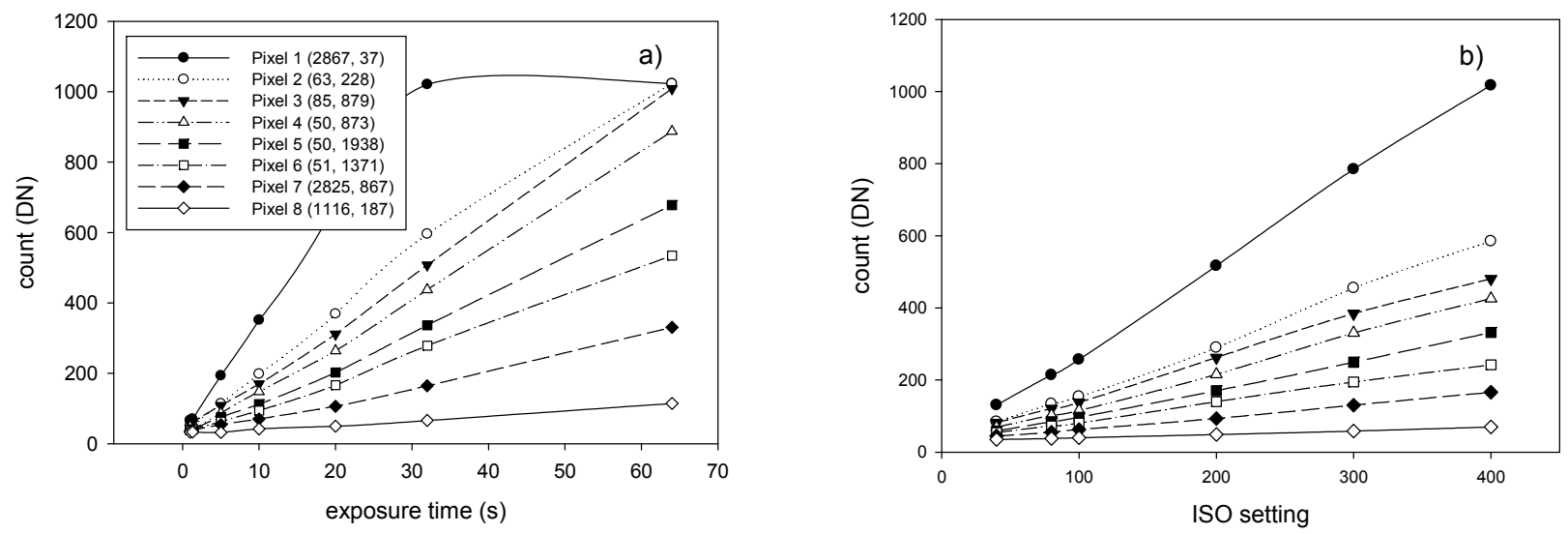

Fig. 6. Dependence of dark current for eight Canon A570 pixels. a) dependence on exposure time. Taken at $24{ }^{\circ} \mathrm{C}$ with $\mathrm{ISO}=400 . \mathrm{b}$ ) dependence on ISO setting. Taken at $24^{\circ} \mathrm{C}$ with an exposure time of $32 \mathrm{~s}$.

Shown in Fig. 7a is the dark current dependence on temperature for the same eight pixels on the A570's imager. Ten frames each were taken at temperatures of $24,30,35,40$, and $46{ }^{\circ} \mathrm{C}$, with an ISO setting of 400 , and an exposure time of $32 \mathrm{~s}$. The camera was heated inside the enclosure to the desired temperature. After configuring the camera to take a series of images, it was placed back in the enclosure with a two minute wait prior to taking exposures. However, inconsistent results were seen in the dark current dependence on temperature. After examining a series of images taken at one setting it became apparent that the dark current increased significantly from frame to frame within a single series of images at identical settings. Shown in Fig. $7 \mathrm{~b}$ is the response of dark current for the same eight pixels versus frame number during a single run. Images were taken at an ISO setting of 400, with an exposure time of $32 \mathrm{~s}$, and at external temperatures ranging from $45.6^{\circ} \mathrm{C}$ for the first frame to $46.6{ }^{\circ} \mathrm{C}$ at the last frame. It is generally held that dark current roughly doubles with an increase in temperature of $8{ }^{\circ} \mathrm{C} .{ }^{8}$ Almost all of the eight pixels had dark current that doubled in the time it took to take the frames depicted, despite the fact that the external temperature increased by only $1.0^{\circ} \mathrm{C}$. Therefore it is likely that the temperature of the imager itself increased significantly more than the external temperature during this run. To obtain a more accurate response to temperature for the A570 pixels it would be necessary to take more care with the conditions of the experiment, possibly by allowing the sensor significantly more time at a given temperature prior to taking any frames. However, it is illustrative to note how significantly the imager was influenced by operation of the camera itself in these series of images even with some means of control over the conditions. If a single dark frame was used to correct another dark frame during this series, it would consistently subtract too many counts. 

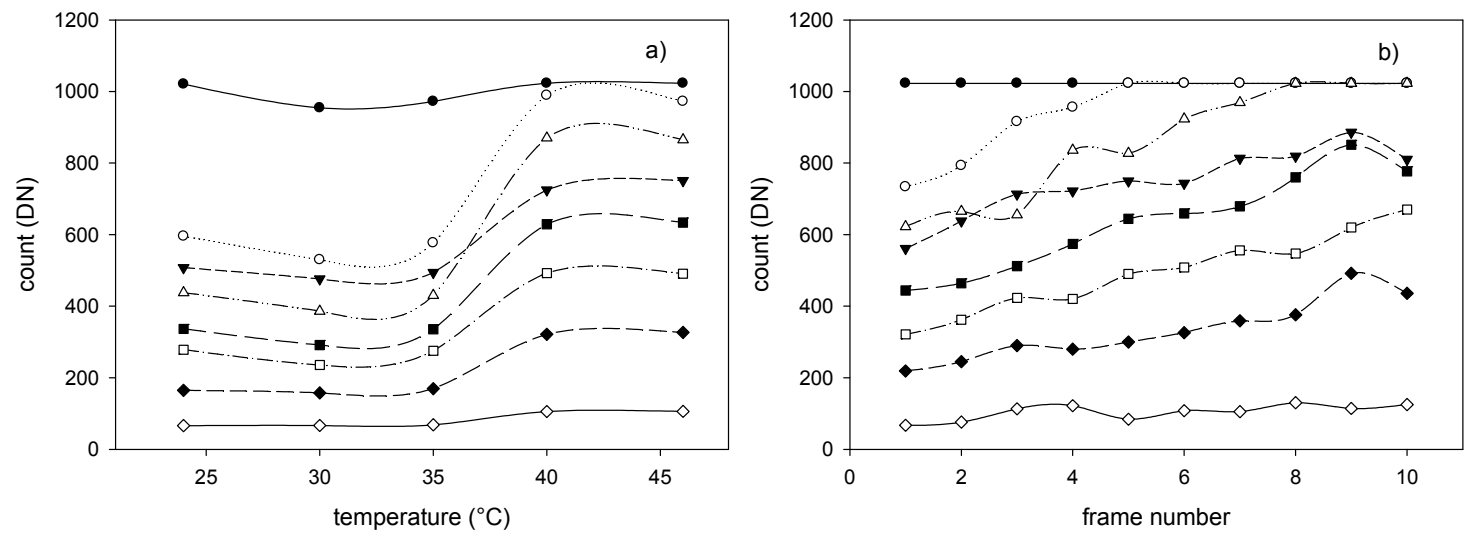

Fig. 7. a) Dependence of dark current for the eight Canon A570 pixels on temperature. Taken with ISO $=400$ with an exposure time of $32 \mathrm{~s}$. b) dependence of dark current for the eight Canon A570 pixels on frame number for a set of ten images taken at ISO $=400,46^{\circ} \mathrm{C}$, and with an exposure time of $32 \mathrm{~s}$ (same pixels as shown in Fig. 6).

\section{METHOD OF CORRECTION FOR DARK CURRENT}

Our method of correction is dependent upon finding the chip's hot pixels, pixels that have higher than average values of dark noise. These hot pixels are unique to every imager and arise due to impurities within the pixel. ${ }^{3}$ To find the hot pixels, a pixel is compared to its neighbors by using the following equation:

$$
\operatorname{hotpix}(x, y)=\sum_{i=-1}^{1} \sum_{j=-1}^{1} \operatorname{weight}(i, j) \cdot \operatorname{pixel}(x+i, y+j),
$$

where $(x, y)$ are the coordinates of the pixel on the image, and pixel $(x, y)$ is its dark count. The weight $(i, j)$ is chosen to be dependent on a neighbor's relative location to the pixel and so that the sum of all weighting factors equals zero. Different weighting factors and number of neighbors can be chosen. For the corrections done in this paper, the center pixel was weighted with a value of 1 , the pixels on the corners were weighted with a value of -0.05 , and the neighbors directly adjacent were weighted with a value of -0.2 . If all neighboring pixels have comparatively similar values to the center pixel, the value for hotpix $(x, y)$ will be close to zero. However, if all neighboring pixels have comparatively smaller values, the value for $\operatorname{hotpix}(x, y)$ will be large. Next the values of hotpix(x,y) are ranked and the $n$ hottest pixels are chosen. The average of hotpix $(x, y)$ for the $n$ hottest pixels is calculated and is used as a dark current indicator for the chip, $D_{\text {ind }}$. The values for $\operatorname{hotpix}(x, y)$ are then fitted as a quadratic least squares function of the indicator such that:

$$
\operatorname{hotpix}(x, y)=a \cdot D_{\text {ind }}^{2}+b \cdot D_{\text {ind }}+c .
$$

Pixels with poor fits are disregarded from the calculation. The values for the fitting parameters $a, b$, and $c$ can then be saved for the hottest pixels along with the coordinates of the hot pixels. The next step in the protocol is to determine the counts of all pixels with respect to $D_{\text {ind }}$. To accomplish this, the counts of each pixel for frames taken with different amounts of dark current, is fitted with a quadratic least squares fit versus the dark current indicator. Since the fit is later used to calculate the dark count, independently from the neighboring pixels, the actual count of the pixels (not the counts with respect to the neighboring pixels) is used to determine the fitting parameters. Therefore, three matrices are calculated and stored with unique $a, b$, and $c$ parameters for every pixel on the imager. These parameters can then be used to evaluate the relative amount of dark current in any image within the calibrated regime.

It was shown that each imager had pixels that demonstrated predictable behavior of pixels on each imager to exposure time, gain, and temperature. These pixels could therefore be used to accurately predict the amount of dark current for all pixels on the imagers. 


\section{DARK CURRENT CORRECTION}

\subsection{Canon PowerShot SD1000}
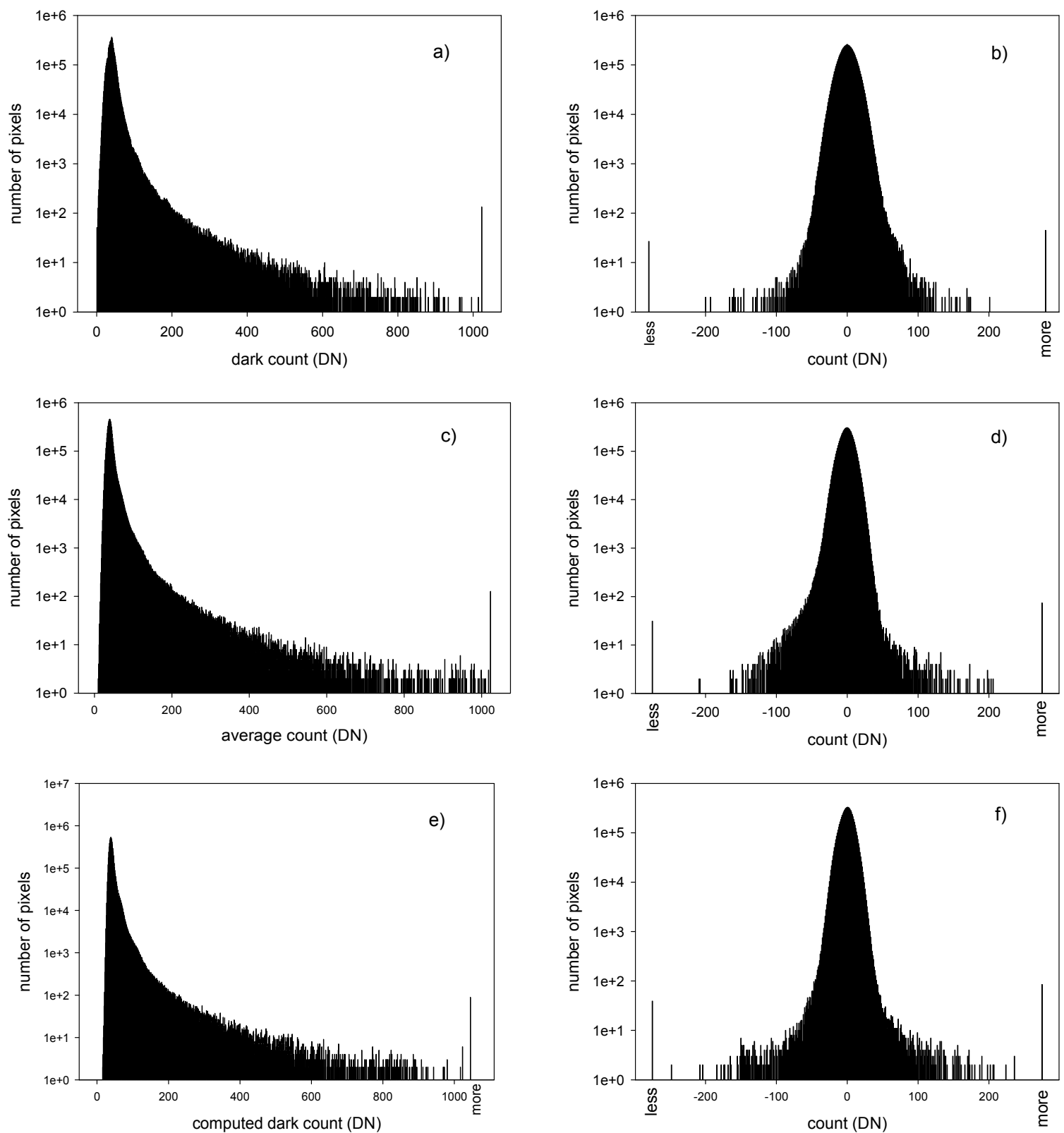

Fig. 8. Correction of a $30 \mathrm{~s}$, ISO $=400,24{ }^{\circ} \mathrm{C}$ image. a) histogram of the uncorrected frame. b) histogram of the corrected frame using an individual frame for correction. c) histogram of the master frame. d) histogram of the corrected frame using the master frame for correction. e) histogram of the computed frame. f) histogram of the corrected frame using the computed frame for correction.

To test the possibility of dark noise correction in the SD1000 using hot pixels as indicators of dark current in an image, we took three dark frames each at $24{ }^{\circ} \mathrm{C}$, with an ISO setting of 400, at 1, 5, 10, 20, 30, 40, 50, and $60 \mathrm{~s}$ exposure times, representing a total of $648 \mathrm{~s}$ of camera time. These frames were used to generate the coefficients of correction. A computed dark frame was generated for a dark frame taken with an exposure time of $30 \mathrm{~s}$, taken at $24^{\circ} \mathrm{C}$, and with an ISO setting of 400 . Correction was done by subtracting the original image from the computed 
image. For comparison, we also used for subtraction both a single dark frame and an averaged master frame using four images. The averaged master frame was generated by taking a pixel by pixel average of the four frames with the maximum and minimum values removed from the average. Shown in Fig. 8 are the histograms for the original image (Fig. 8a), the corrected frame after subtraction of a single dark frame (Fig. 8b), the averaged master frame (Fig. 8c), the corrected frame after subtraction of the averaged master frame (Fig. 8d), the computed dark frame (Fig. 8e), and the corrected frame after subtraction of the computed frame (Fig. 8f).

To test the relative success of correction we calculated the standard deviation of the original frame and the corrected frames. In an ideal correction, subtraction of a second dark frame would yield a histogram with a narrow distribution around the value of zero with only small deviations due to shot noise, the noise due to the statistical nature of the generation of dark current. A smaller standard deviation of the corrected dark frame would indicate a better correction than one with a larger standard deviation. In addition, a large standard deviation indicates a dark frame with large variations in the uniformity of dark noise. It is of note though that using the standard deviation as the sole means of testing the quality of correction is not adequate because it fails to take into account the effect small numbers of outlier pixels with poor correction would have on the quality of the image. These pixels would have values greatly deviating from the dark noise in the original image and therefore a correction would result in these pixels showing up as either darker than the surroundings due to an inaccurate over-correction, or showing up as much brighter than the surroundings due to an inaccurate under-correction. Ultimately these pixels do need to be properly corrected to obtain the most aesthetically pleasing image, however the standard deviation provides an easily comparable value that can be used to approximately weigh the success of correction. The histogram of the uncorrected frame shows a single sharp peak with low dark current values and a steady decrease in frequency of pixels at higher dark current values. This trend continues until saturation with about 135 reading saturation. This histogram has a standard deviation of 18.6 counts. Standard deviations of the corrected frames are 12.0, 10.1, and 9.5 counts for corrections using the single frame, the averaged master frame, and the computed frame respectively. Thus the correction using the computed frame has a $49 \%$ decrease in the standard deviation compared to the original, and shows an improvement over the use of both the single frame or the averaged master frame corrections.
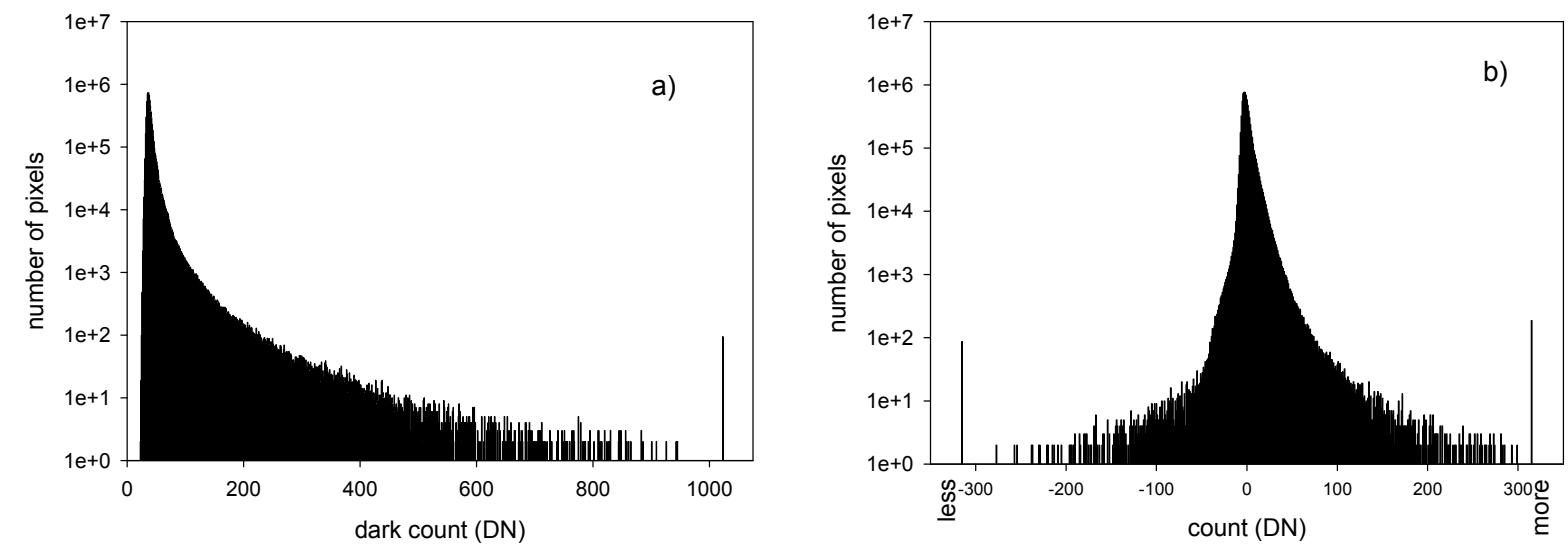

Fig. 9. Correction of a $64 \mathrm{~s}, \mathrm{ISO}=100,36{ }^{\circ} \mathrm{C}$ image. a) histogram of the uncorrected frame. b) histogram of the corrected frame using a computed frame for correction.

An advantage of using the computational method of correction is that it does not require dark frames to be taken at the same time as the original image with the same exact settings. To show that the computational method can work on dark frames not taken with any of the same settings as the dark frames used to generate the coefficients of correction, a computed frame was generated for a dark frame taken at $64 \mathrm{~s}$, with an ISO setting of 100, and at an external temperature of $36^{\circ} \mathrm{C}$. The histogram for the original frame has a standard deviation of 16.8 counts and is displayed in Fig. 9a. The histogram for the corrected frame after subtraction of the computed frame is shown in Fig. 9b. This histogram has a standard deviation of 7.8 counts, a decrease of $54 \%$ from the original. 


\subsection{Canon S2IS}

To demonstrate for the S2IS that dark frames can be computed for varying temperature, we varied the temperature to obtain frames with varying levels of dark current and calculated the coefficients of correction. To determine the coefficients, 5 dark frames were taken at each $30,35,40$, and $45^{\circ} \mathrm{C}$, with exposure times of $51 \mathrm{~s}$, and with an ISO setting of 200, representing $1020 \mathrm{~s}$ of camera time. The dark current correction was applied to an image taken at $35^{\circ} \mathrm{C}$, ISO setting of 200, and an exposure time of $51 \mathrm{~s}$. The original frame, shown in Fig. 10a, has a histogram with a standard deviation of 19.5 counts. This histogram shows two distinct peaks, possibly due to the presence of specific metal defects. ${ }^{9}$ The histogram for correction using a single frame is displayed in Fig. $10 \mathrm{~b}$ and has a standard
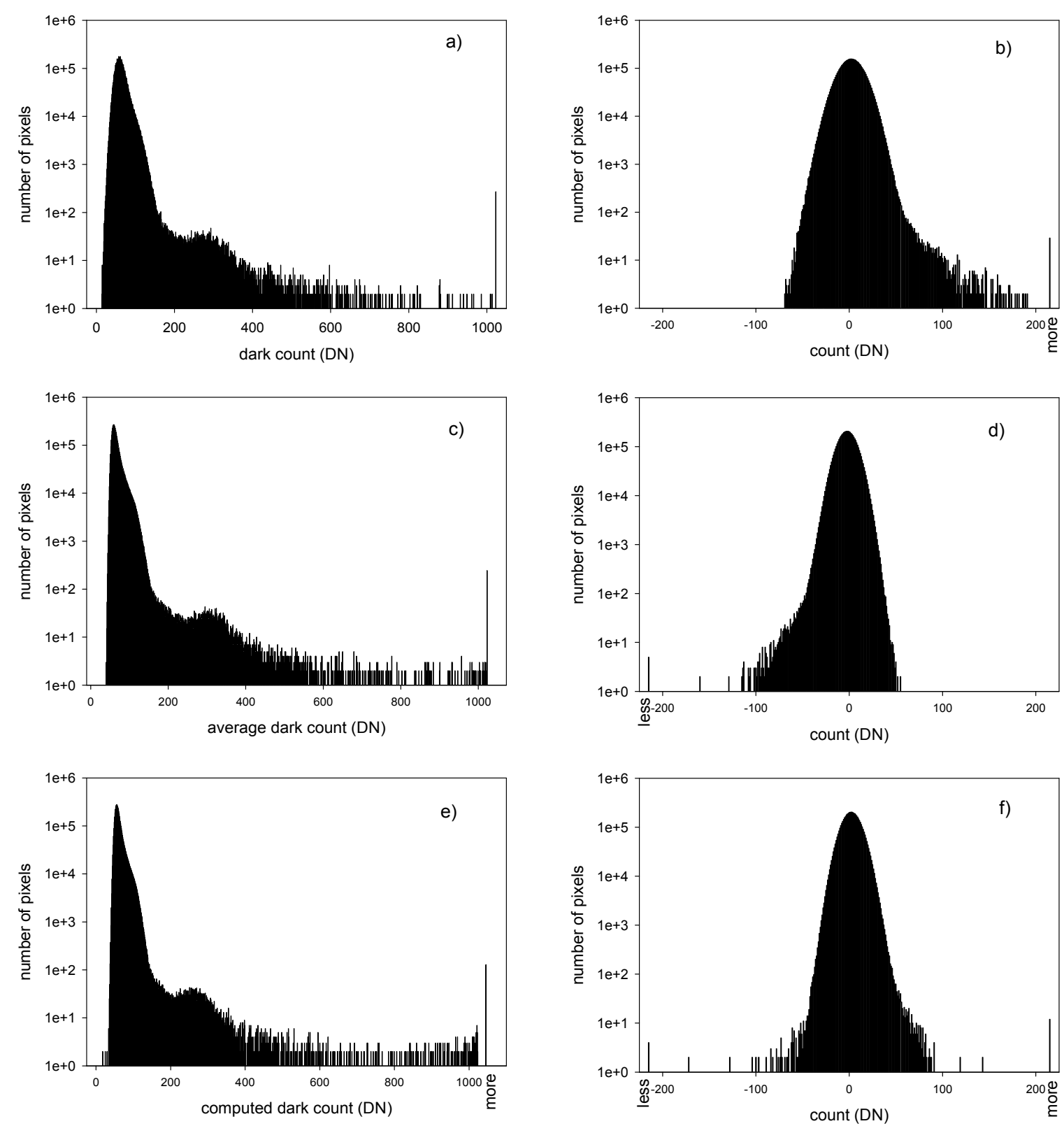

Fig. 10. Correction of a $51 \mathrm{~s}$, ISO $=200,35{ }^{\circ} \mathrm{C}$ image. a) histogram of the uncorrected frame. b) histogram of the corrected frame using an individual frame for correction. c) histogram of the master frame. d) histogram of the corrected frame using the averaged frame for correction. e) histogram of the computed frame. f) histogram of the corrected frame using the computed frame for correction. 
deviation of 13.3 counts. This histogram shows a distinct irregularity between the two sides of the histogram with more pixels showing a positive value after correction than before. This is likely due to the single frame used for correction being taken before the original frame and therefore having less dark current. An averaged master frame was generated using 10 frames, with 5 frames taken before the original frame and 5 taken after that frame. The histogram for the averaged master frame is shown in Fig. 10c, and the histogram for correction using this frame is shown in Fig. 10d. This histogram has a standard deviation of 10.1 counts. The computed dark frame is depicted in Fig. 10e, and the histogram of the corrected frame is displayed in Fig. 10f. This corrected frame has a standard deviation of 10.3 counts. Thus, the corrected histogram using the computed frame has a $47 \%$ reduction in the standard deviation as compared to the original. The computational method of correction gives a similar result to that of the averaged master frame and shows an improvement of correction using the single dark frame.

\subsection{Canon PowerShot A570}

Five images were taken with the A570 IS at each 1, 2.5, 5, 10, 20,32, and $64 \mathrm{~s}$, with an ISO setting of 400, and at a temperature of $24{ }^{\circ} \mathrm{C}$, representing a total of $672.5 \mathrm{~s}$ of camera time. The original frame is shown in Fig. 11a. Like the SD1000, it has a single peak at low dark current values that declines in pixel frequency as dark count increases. However, this camera has significantly more pixels with higher dark count values than the SD1000 has at similar settings, suggesting the presence of more impurities or defects. The histogram of the original frame has a standard deviation of 40.6 counts. The histogram for the correction using the single frame is shown in Fig. 11b and has a standard deviation of 13.4 counts. The histogram for the averaged master dark frame, consisting of an average using nine frames, is shown in Fig. 11c. The histogram for the corrected frame using the master frame is shown in Fig. 11d. This histogram has a standard deviation of 11.4 counts and shows a slight bulge in the peak on the side of positive values that is consistent with the fact that the frames comprising the average were taken prior to the original frame. The histogram for the computed dark frame is shown in Fig 11e. The histogram for the corrected frame using the computed frame is shown in Fig. 11f. This histogram also has a standard deviation of 11.4 counts, a $72 \%$ decrease in the standard deviation of the original. The correction using the computed frame has a similar standard deviation as the corrected frame using the averaged master frame, and also shows an improvement over using single frame correction.

\section{SUMMARY}

Dark current within consumer cameras has been shown to vary with exposure time, temperature, and ISO setting. In addition, operation of the camera itself has been shown to alter the amount of dark current in subsequent frames, likely due to an increase in temperature of the imager itself from heat generated by internal camera circuitry. Correction for dark current has been shown to be successful despite this added complexity. Correction using single dark frames, averaged master frames, and computed dark frames using hot pixels as dark current indicators show improvement in the levels of noise compared to the original frames. In particular, the method of using past dark current behavior and hot pixels on the imager as indicators to compute dark frames has been shown to be comparable, and at times better, than the use of an averaged master frame, as well as being consistently more accurate than the use of a single dark frame, the apparent method of choice designed to be used by most consumer cameras. Correction using computed frames show a $49 \%$ to $72 \%$ decrease in the amount of noise compared to the originals. Computational dark frame correction also has the benefit of being possible at any time after an image is taken and does not require dark frames taken at the identical temperature, ISO setting, and exposure time as the original. 

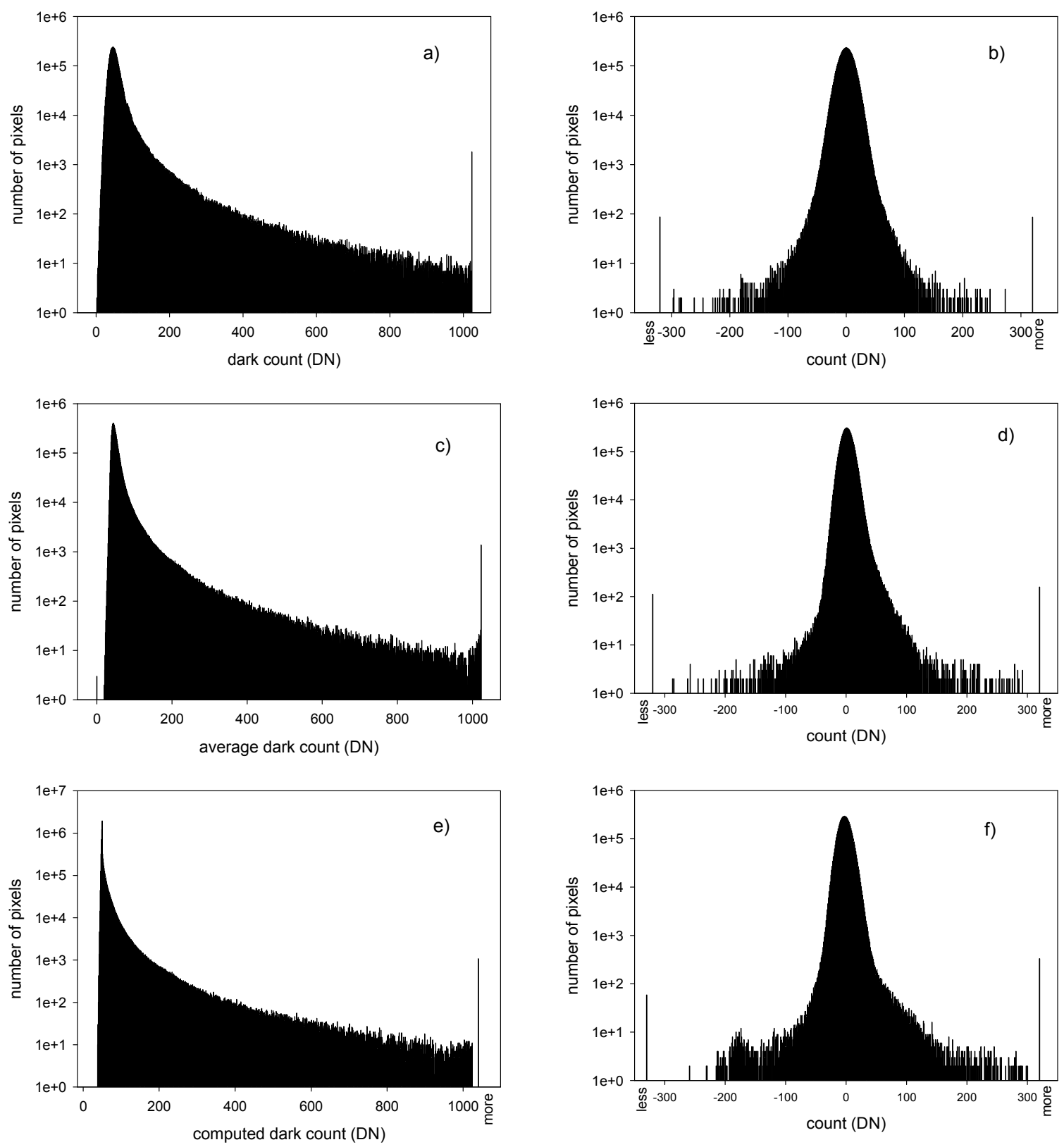

Fig. 11. Correction of a $32 \mathrm{~s}$, ISO $=400,24{ }^{\circ} \mathrm{C}$ image. a) histogram of the uncorrected frame. b) histogram of the corrected frame using an individual frame for correction. c) histogram of the master frame. d) histogram of the corrected frame using the averaged frame for correction. e) histogram of the computed frame. f) histogram of the corrected frame using the computed frame for correction.

\section{ACKNOWLEDGEMENTS}

The authors would like to acknowledge financial support by PSU Faculty Development Grant. 


\section{REFERENCES}

[1] Widenhorn, R., Rest, A., Blouke, M. M., Berry, R. L., and Bodegom, E., "Computation of dark frames in digital imagers," Proc. of SPIE. 6501, 650103 (2007)

[2] Porter, W.C., Kopp, B., Dunlap, J.C., Widenhorn, R., and Bodegom, E.. "Dark current measurements in a CMOS imager," Proc. of SPIE. 6816, 68160C (2008)

[3] Dunlap, J.C., Sostin, O., Widenhorn, R., and Bodegom, E., "Dark current behavior in DSLR cameras," Proc. of SPIE 7249, 72490N (2009)

[4] S.M. Sze, "Physics of Semiconductor Devices," (John Wiley \& Sons, 1967)

[5] McGrath, R.D., Doty, J., Lupino, G., Ricker, G., and Vallerga, J., "Counting of deep-level traps using a charge-coupled device," IEEE Trans. Electron Devices, vol. ED-34, 2555, (1987)

[6] McColgin, W.C., Lavine, J.P., Kyan, J., Nichols, D.N., and Stancampiano, C.V., "Dark current quantization in CCD image sensors," International Electron Device Meeting 1992, p. 113, 13-16 (1992)

[7] CHDK Wiki members, "CHDK Wiki", http://chdk.wikia.com/wiki/CHDK

[8] Theuwissen, A.J.P, "Solid-State Imaging with Charge-Coupled Devices," Kluwer Academic Publishers, (1995)

[9] McColgin, W.C., Lavine, J.P., and Stancampiano, C.V. "Probing metal defects in CCD image sensors," Proc. of Mat. Res. Soc. Symp. 378, 713 (1995) 